\title{
Noise Figure Characteristics of Erbium Doped Fiber Amplifiers at single and multiple amplified stage
}

\author{
Dr. Ragheed M. Ibrahim \\ Department of Physics \\ College of Education \\ University of Mosul
}

\author{
Nawzat S. Saadi \\ Department of Physics \\ College of Science \\ University of Duhok
}

\section{\& \\ Saad F. Ramadhan \\ Department of Physics \\ College of Science \\ University of Duhok}

Received
$01 / 02 / 2011$
Accepted

06 / 04 / 2011

\begin{abstract}
(الملخص
تعرض هذه الدراسة الحسابات النظرية لمكبر الليف الضوئي المطعم بأيونات الأربيوم

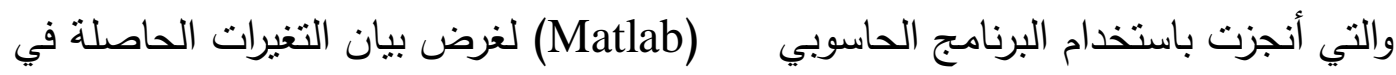

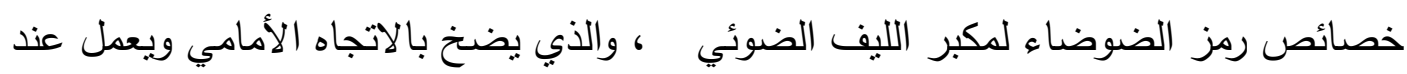

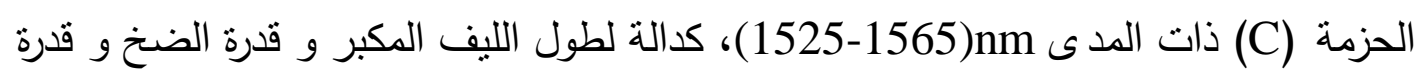

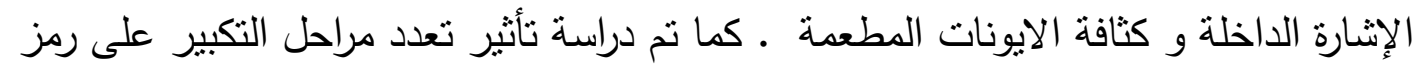

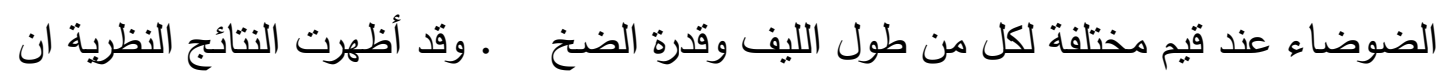

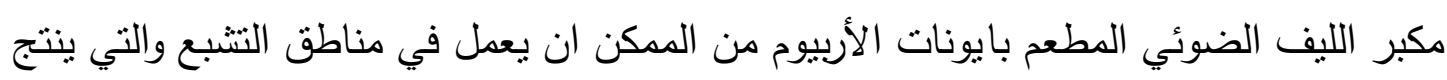

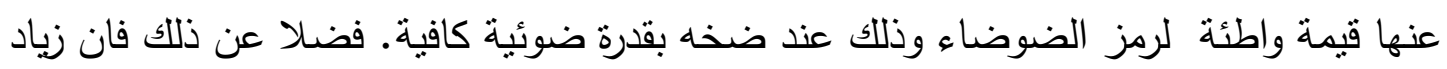

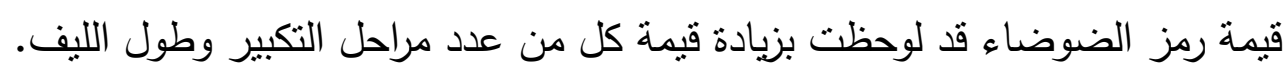

\begin{abstract}
In this study, an Erbium Doped Fiber Amplifier (EDFAs) simulation program has been written in Matlab to characterize Noise Figure variations of a forward pumped EDFAs operating in $\mathrm{C}$ band (1525-1565 nm) as functions of $\mathrm{Er}^{3+}$ fiber length, injected pump power, signal input power and $\mathrm{Er}^{3+}$ doping density. The effect of multiple amplified stage on the noise figure was student at different Fiber length
\end{abstract}


and pumping power. The numerical results shows that EDFAs could be operated in saturation regimes leading minimum noise figure when the EDFAs is supplied with sufficient pump power, Moreover a continuous increasing of the noise figure value was demonstrated by increasing all of amplified stage number and fiber length.

\section{I- Introduction}

One of the most important factors limiting the transmission distance in fiber optical communication systems is the optical power loss caused by scattering and absorption mechanisms in optical fiber. Electrical repeaters, which require optical-electrical signal conversion, have previously been used to compensate the power losses increasing with distance. The use of such repeaters in optical communication systems have made the systems more complex and increased their installation $\operatorname{costs}^{[1,2]}$. The invention of the Erbium Doped Fiber Amplifier (EDFAs) in the late eighties was one of the major events in the history of optical communications. It provided new life to the optical fiber transmission window centered at $1550 \mathrm{~nm}$ and the consequent research into technologies that allow high bit-rate transmission over long distances. High bit-rates were also possible with the aid of different dispersion compensation schemes ${ }^{[3]}$.

EDFAs made by doping the silica fiber with erbium ions can operate in a broad range within the $1550 \mathrm{~nm}$ window at which the attenuation of silica fiber is minimum and therefore it is ideal for the optical fiber communication systems operating at this wavelength range. According to the research performed in recent years, it is known that the pumping of erbium doped fiber at $980 \mathrm{~nm}$ or $1480 \mathrm{~nm}$ is the most efficient way. High gain (30 50 dB), large bandwidth $(\geq 90 \mathrm{~nm}$ ), high output power $(10 \sim 20 \mathrm{dBm})$ and low noise figure $(\mathrm{NF}=3 \sim 5 \mathrm{~dB})$ can be obtained using an erbium doped fiber amplifier optimised for $1.55 \mu \mathrm{m}$ range ${ }^{[4]}$.

The principal source of noise in EDFAs is Amplified Spontaneous Emission (ASE), which has a spectrum approximately the same as the gain spectrum of the amplifier. As well as decaying via stimulated emission, electrons in the upper energy level can also decay by spontaneous emission, which occurs at random, depending upon the silica structure and inversion level. Those photons from spontaneous emission may also interact with the dopant ions, and the initial spontaneous emission is therefore amplified in the same manner as the signals, hence becomes (ASE). The noise will propagate with the signal (Amplified Stimulated Emission) to the receiver and cause a degradation of the system performance ${ }^{[2,5,6]}$. EDFAs are currently attracting increased interest, especially for use in optical communication wavelength-division 
multiplexed (WDM) systems. The main reason is the possibility of compensating for optical fiber losses in broad wavelength ranges and developing a large-capacity, long-distance transmission system. The performance of the transmission system is strongly influenced by the gain and noise of used EDFAs. Therefore, detailed information about these EDFAs characteristics is the key for advanced design of WDM systems $^{[3,7]}$.

\section{II- EDFAs Configuration}

The basic configuration of EDFAs is shown in figure (1). EDFAs consist of optical isolators, Wavelength Division Multiplexer (WDM) coupler to combine pump and signal lights, active fiber that consist of a short section of fiber where the core of a silica fiber is doped with trivalent Erbium ions $\left(\mathrm{Er}^{+3}\right)$, forward pumping lasers to pump the erbium ions into higher energy levels and achieve population inversion.

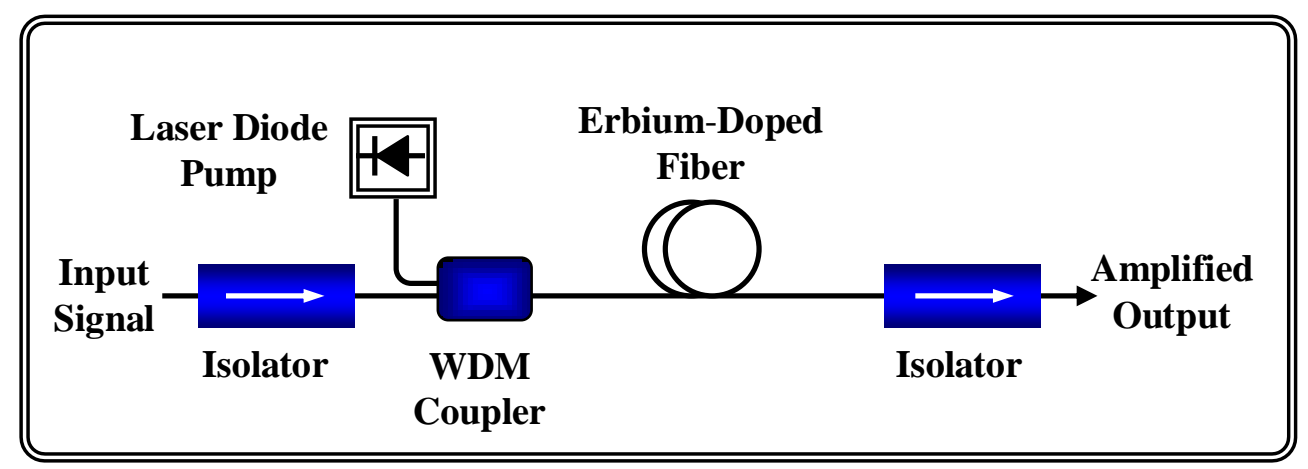

Figure (1): Forward pumped EDFA structure

The gain characteristics of EDFAs depend mainly on their pumping schemes. EDFAs can be pumped at $980 \mathrm{~nm}$ or $1480 \mathrm{~nm}$, and with different configurations: backward pumping, forward pumping or dual (bi-directional) pumping. In forward pumping, both of the signal and pump lights propagate in the same direction through the fiber whereas in the backward pumping they propagate in the opposite direction. The forward pumping direction provides the lowest noise figure. In fact, the noise is sensitive to the gain and the gain is the highest when the input power is the lowest. Backward pumping provides the highest saturated output power. dual (bi-directional) pumping ,combines the two schemes, has a higher performance than the other two by combining the lowest noise figure and the highest output power advantageous although it requires two pump lasers ${ }^{[5,6,8]}$.

\section{III- Theoretical part}

A common way to characterize the performance of a preamplifier is through its noise figure. The noise figure (NF) is defined as the ratio of 
the signal to noise ratio at the input of the preamplifier-receiver to that at the output of the preamplifier-receiver combination ${ }^{[4]}$

$$
\mathrm{NF}=\frac{(\mathrm{SNR})_{\text {in }}}{(\mathrm{SNR})_{\text {out }}}
$$

The noise figure will always be greater than one, due to the fact that the amplifier adds noise during the amplification process and the signal to noise ratio at the output is always lower than that at the input. The noise figure value is usually given in $\mathrm{dB}$. A high noise figure implies that the signal to noise ratio has been impaired by the amplification process. One can show that for an ideal amplifier the quantum limit of the noise figure i.e., the best value achievable is $3 \mathrm{~dB}$. Noise figures close to $3 \mathrm{~dB}$ have been obtained with erbium-doped fiber amplifiers.

The noise figure at the input of the amplifier is computed assuming that the signal is shot noise limited. The shot noise for a current $I_{s}$ in a electrical bandwidth $B_{e}$ is $2 \mathrm{e}_{\mathrm{s}} \mathrm{B}_{\mathrm{e}}$, where the current $I_{\mathrm{s}}$ is related to the optical power $\mathrm{P}$ by the relation $\mathrm{I}_{\mathrm{s}}=(\mathrm{P} / \mathrm{h} v) \mathrm{e}$. The electrical signal to noise ratio at the input of the amplifier is thus ${ }^{[1]}$.

$$
(\mathrm{SNR})_{\text {in }}=\frac{\mathrm{I}_{\mathrm{s}}^{2}}{2 \mathrm{eI}_{\mathrm{s}} \mathrm{B}_{\mathrm{e}}}=\frac{\mathrm{I}_{\mathrm{s}}}{2 \mathrm{eB}_{\mathrm{e}}}
$$

The signal to noise ratio at the output of the amplifier is given by the expression

$$
(\mathrm{SNR})_{\text {out }}=\frac{\left(\mathrm{GI}_{\mathrm{s}}\right)^{2}}{\mathrm{~N}_{\mathrm{s}-\mathrm{sp}}+\mathrm{N}_{\mathrm{sp}-\mathrm{sp}}+\mathrm{N}_{\text {shot }}}
$$

Where $\mathrm{N}_{\mathrm{s} \text {-sp }}$ is the power spectrum of the signal-spontaneous beat noise, $\mathrm{N}_{\text {sp-sp }}$ is the power spectrum of the spontaneous-spontaneous beat noise and $\mathrm{N}_{\text {shot }}$ is the shot noise.

(the thermal noise will be negligible at high enough gains). The noise figure can then be calculated to be:

$$
\mathrm{NF}=\frac{\mathrm{G} \frac{\mathrm{I}_{\mathrm{s}}^{2}}{\mathrm{e}} \mathrm{I}_{\mathrm{sp}} \frac{1}{\mathrm{~B}_{0}}+\frac{1}{4} \frac{\mathrm{I}_{\mathrm{s}}}{\mathrm{e}} \mathrm{I}_{\mathrm{sp}}^{2} \frac{\left(2 \mathrm{~B}_{\mathrm{o}}-\mathrm{B}_{\mathrm{e}}\right)}{\mathrm{B}_{\mathrm{o}}^{2}}+\mathrm{I}_{\mathrm{s}}\left(\mathrm{GI}_{\mathrm{s}}+\mathrm{I}_{\mathrm{sp}}\right)}{\left(\mathrm{GI}_{\mathrm{s}}\right)^{2}}
$$

Using the fact that the spontaneous emission power current is given by $\mathrm{I}_{\mathrm{sp}}=2 \mathrm{n}_{\mathrm{sp}}(\mathrm{G}-1) \mathrm{B}_{\mathrm{o}}$, we can further write the noise figure as

$$
N F=2 n_{s p} \frac{(G-1)}{G}+\frac{1}{G}+\frac{n_{s p}(G-1)^{2} e\left(2 B_{o}-B_{e}\right)}{G^{2} I_{s}}+\frac{2(G-1) n_{s p} e B_{o}}{G^{2} I_{s}}
$$

Where $B_{o}$ is the optical bandwidth and $n_{s p}$ is the inversion parameter. 
The first term is the dominant term in the right-hand side of equation (5) Assuming that $G \gg 1$ and reasonably high input powers, the noise figure is given by $2 n_{s p}$. Since $n_{s p}$ is at best 1 , when complete inversion is attained, the lowest noise figure achievable is 2 (i.e. in $\mathrm{dB}$ unit equal $3 \mathrm{~dB}$ ), given the assumptions just outlined. This is the source of the often quoted $3 \mathrm{~dB}$ noise figure limit. Cases where the noise figure is below the $3 \mathrm{~dB}$ noise limit can sometimes be encountered. They usually occur when the gain is low and the inversion factor $\mathrm{n}_{\mathrm{sp}}$ is close to unity, a situation that typically arises when the fiber length is short. Since most practical systems are either high gain or else not well inverted because of saturation, this situation is seldom encountered in practice. The $\mathrm{n}_{\mathrm{sp}}$ depends on the inversion in the amplifier as well as the absorption cross sections $\sigma_{\mathrm{a}}(\lambda)$ and emission cross sections $\sigma_{\mathrm{e}}(\lambda)$ at the signal wavelength $\lambda^{[1,3]}$

$$
\mathrm{n}_{\mathrm{sp}}=\frac{\sigma_{\mathrm{e}}(\lambda) \mathrm{N}_{2}}{\sigma_{\mathrm{e}}(\lambda) \mathrm{N}_{2}-\sigma_{\mathrm{a}}(\lambda) \mathrm{N}_{1}}=\frac{\mathrm{N}_{2}}{\mathrm{~N}_{2}-\frac{\sigma_{\mathrm{a}}(\lambda)}{\sigma_{\mathrm{e}}(\lambda)} \mathrm{N}_{1}}
$$

Where $\mathrm{N}_{1}$ is the population of ground state and $\mathrm{N}_{2}$ is the population of excited state. The noise figure can also be very simply written in terms of the ASE power exiting the fiber in bandwidth of the EDFAs $\Delta v$. Considering the first two terms of equation (5) since the noise power is given by $\mathrm{P}_{\mathrm{ASE}}=2 \mathrm{n}_{\mathrm{sp}} \mathrm{h} v \Delta v(\mathrm{G}-1)$ we can write ${ }^{[4]}$

$$
\mathrm{NF}=\frac{\mathrm{P}_{\mathrm{ASE}}}{\mathrm{h} v \Delta v \mathrm{G}}+\frac{1}{\mathrm{G}}
$$

As it can be seen from (7), EDFAs noise figure depends directly on forward $\mathrm{P}_{\mathrm{ASE}}$ and gain. Noise Figure increases with increasing $\mathrm{P}_{\mathrm{ASE}}$, on the other hand, decreases with increasing gain ${ }^{[1,6]}$.

The noise figure for a system consisting of a chain of optical amplifiers can be computed from the noise figure for an individual amplifier ${ }^{[9]}$. Consider a system of $\mathrm{N}$ amplifiers where $\mathrm{SNR}_{\mathrm{i}}$ denotes the signal to noise ratio after amplifier (i), and each amplifier provides a gain $\mathrm{G}$ to exactly compensate the span loss $\mathrm{L}$. The overall noise figure of the system is given by:

$$
\mathrm{NF}_{\mathrm{sys}}=\frac{\mathrm{SNR}_{0}}{\mathrm{SNR}_{\mathrm{N}}}=\frac{\mathrm{SNR}_{0}}{\mathrm{SNR}_{1}} \frac{\mathrm{SNR}_{1}}{\mathrm{SNR}_{2}} \cdots \cdots \frac{\mathrm{SNR}_{\mathrm{N}-1}}{\mathrm{SNR}_{\mathrm{N}}}
$$

Where $\mathrm{SNR}_{0}$ is the signal to noise ratio at the input of the system (immediately after the transmitter, and prior to the first span of fiber). The SNR ratios in equation (8) are the noise figures of each amplifier multiplied by $1 / \mathrm{L}$ since the amplifier noise figure is defined by the SNR's 
immediately prior and after the amplifier. In equation (8), each $\mathrm{SNR}_{\mathrm{i}}$ is separated from the following amplifier by a span with loss L, hence we obtain for the system noise figure, in logarithmic units ${ }^{[5]}$

$$
\mathrm{NF}_{\mathrm{sys}}=\mathrm{G} \mathrm{NF}+\mathrm{G} \mathrm{NF}+\cdots \cdots+\mathrm{G} \mathrm{NF}_{\mathrm{N}}
$$

assuming all the amplifiers have an equal noise figure and $\mathrm{G}=\mathrm{L}$. The SNR degradation in a cascaded amplifier transparent chain is seen to be linear with the number of amplifiers.

An interesting result can be derived when $\mathrm{G}$ and $\mathrm{L}$ are different, as is the case for a multistage amplifier constructed by piecing together several amplifiers. Equation (8) is then written more generally as ${ }^{[9]}$

$$
\mathrm{NF}_{\mathrm{sys}}=\frac{\mathrm{NF}_{1}}{\mathrm{~L}_{1}}+\frac{\mathrm{NF}_{2}}{\mathrm{~L}_{1} \mathrm{G}_{1} \mathrm{~L}_{2}}+\cdots \cdots+\frac{\mathrm{NF}_{\mathrm{N}}}{\mathrm{L}_{1} \mathrm{G}_{1} \mathrm{~L}_{2} \mathrm{G}_{2} \cdots \cdots \mathrm{L}_{\mathrm{N}}}
$$

where $L_{i}$ and $G_{i}$ refer to the loss prior to amplifier $i$ and the gain of amplifier $i$, respectively. With $L_{i}=1$ for all (i) this becomes

$$
\mathrm{NF}_{\text {sys }}=\mathrm{NF}_{1}+\frac{\mathrm{NF}_{2}}{\mathrm{G}_{1}}+\cdots \cdots+\frac{\mathrm{NF}_{\mathrm{N}}}{\mathrm{G}_{1} \mathrm{G}_{2} \cdots \cdot \mathrm{G}_{\mathrm{N}-1}}
$$

which shows that the noise figure of a multi-stage amplifier is dominated by the noise of the first stage ${ }^{[6,9]}$.

\section{IV- Results and Discussion}

In this study, the rate and propagation equations characterizing a forward pumped $\mathrm{C}$ band ,from about (1525 to 1565$) \mathrm{nm}$, EDFAs were numerically solved. Noise figure can be obtained as a function of four fundamental fiber parameters namely: fiber length, pump power, signal input power and erbium doping density.

Table (1) shows the typical EDFAs parameters used in numerical calculations.

Table (1): The typical EDFAs parameters used in numerical calculations ${ }^{[3]}$.

\begin{tabular}{||l|c|c||}
\hline \multicolumn{1}{|c|}{ Parameter } & Value & Unit \\
\hline \hline Core Radius & 2.2 & $\mu \mathrm{m}$ \\
\hline Erbium Doping Radius & 2.2 & $\mu \mathrm{m}$ \\
\hline Erbium Metastable Lifetime & 10 & $\mathrm{~ms}$ \\
\hline Numerical Aperture & 0.24 & ------ \\
\hline Erbium Ion Density & 150 & $\mathrm{ppm}$ \\
\hline Noise Bandwidth & 104.19 & $\mathrm{~nm}$ \\
\hline Pump Wavelength & 980 & $\mathrm{~nm}$ \\
\hline Signal Wavelength & 1550 & $\mathrm{~nm}$ \\
\hline Pump Absorption Cross Section & $1.8 \times 10^{-25}$ & $\mathrm{~m}^{2}$ \\
\hline Signal Absorption Cross Section & $2.14 \times 10^{-25}$ & $\mathrm{~m}^{2}$ \\
\hline Pump Emission Cross Section & $3.15 \times 10^{-25}$ & $\mathrm{~m}^{2}$ \\
\hline
\end{tabular}




\section{1- Single amplified Stage}

The variation of noise figure as a function of fiber length is shown in figure (2) for different pumping powers at a constant signal input power and erbium ion density. In this simulation, seven different pump powers were applied $(10,20,30,40,60,70,80) \mathrm{mW}$ with $150 \mathrm{ppm}$ doping density and using an input signal power of $-30 \mathrm{dBm}$. For a pump power of $30 \mathrm{~mW}$, the increase in noise figure from $85 \mathrm{~m}$ fiber length can be clearly noticed. The reason for this increase is the decreasing gain with sharp pump depletion.

Figure (3) shows the noise figure variations as a function of pump power for different fiber lengths at a constant signal input power and doping density. In the simulation performed for seven different fiber length $(10,20,30,40,60,70,80) \mathrm{m}$, a $-30 \mathrm{dBm}$ signal input power was applied to an EDFAs with an erbium doping rate of $150 \mathrm{ppm}$ and the pump power was increased from (5 $\mathrm{mW}$ to $100 \mathrm{~mW}$ ). In an amplifier having these parameters, it can be seen from the graph that the noise figure decreases with increasing pump power. The high gain in an active fiber with the total population inversion provided causes the spontaneous emission to stay in low levels. The noise figure of the EDFAs varies linearly with ASE power and inversely with the amplifier gain. Therefore, the noise figure of an EDFAs can be reduced to a minimum level by increasing the gain.

The $\mathrm{Er}^{3+}$-ion doping of the fiber will have an influence on the amplifier's characteristics. If the doping level is low, the ground state may become depleted if the number of available ions is fewer than the pump photons ${ }^{[2]}$. The variation of noise figure as a function of erbium ion density is given in figure (4) for a constant fiber length and signal input power. This graph was obtained by using a $10 \mathrm{~m}$ long erbium doped fiber and $-30 \mathrm{dBm}$ signal input power with seven different pump powers were applied $(5,10,20,40,60,80,100) \mathrm{mW}$. From figure (4) it is seen that, noise figure remains constant from approximately $30 \mathrm{ppm}$ even if the pumping power would be increased. Beyond $30 \mathrm{ppm}$ and for a $10 \mathrm{~mW}$ pumping power, insufficient pumping occurs and the noise figure sharply increases due to not to have population inversion.

In figure (5) the variation of noise figure is given as a function of signal input power for a constant fiber length and erbium ion density. In this figure, a $10 \mathrm{~m}$ long EDFAs with an erbium ion density of $150 \mathrm{ppm}$ and six different pump powers $(10,20,40,60,80,100) \mathrm{mW}$ was used. The graph shows that the noise figure of the EDFAs increases with increasing signal input power. The reasons of this return to gain decreases with increasing signal input power because easier saturation of the EDFAs at higher signal powers for a constant pump power ${ }^{[6]}$. 
Noise Figure Characteristics of Erbium Doped Fiber Amplifiers at single and ...

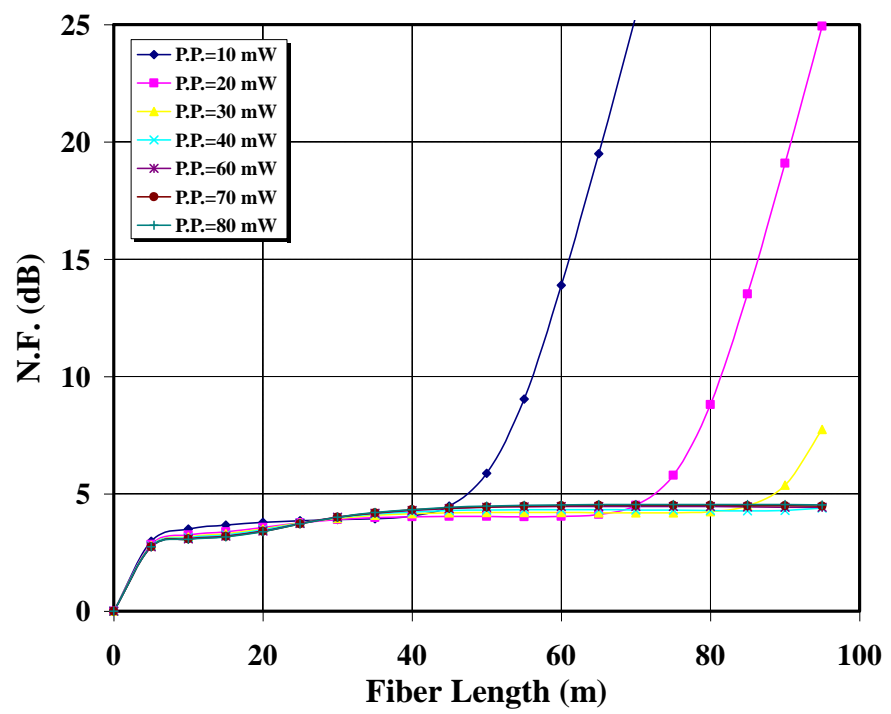

Figure (2): The variation of noise figure versus Fiber Length for different pumping power

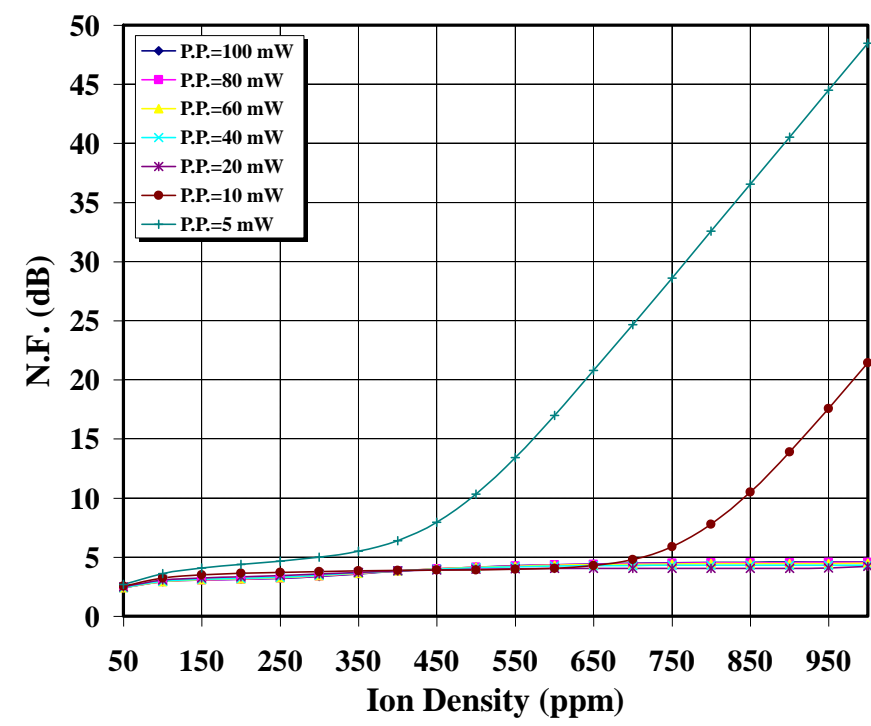

Figure (4): The variation of noise figure versus Ion Density for different pumping power

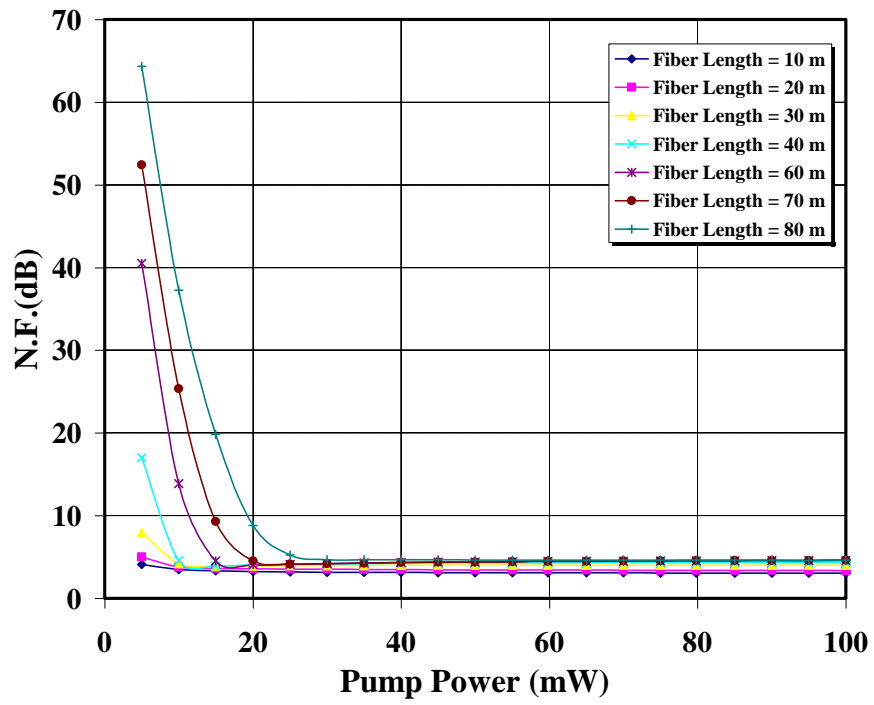

Figure (3): The variation of noise figure versus Pump power for different fiber length

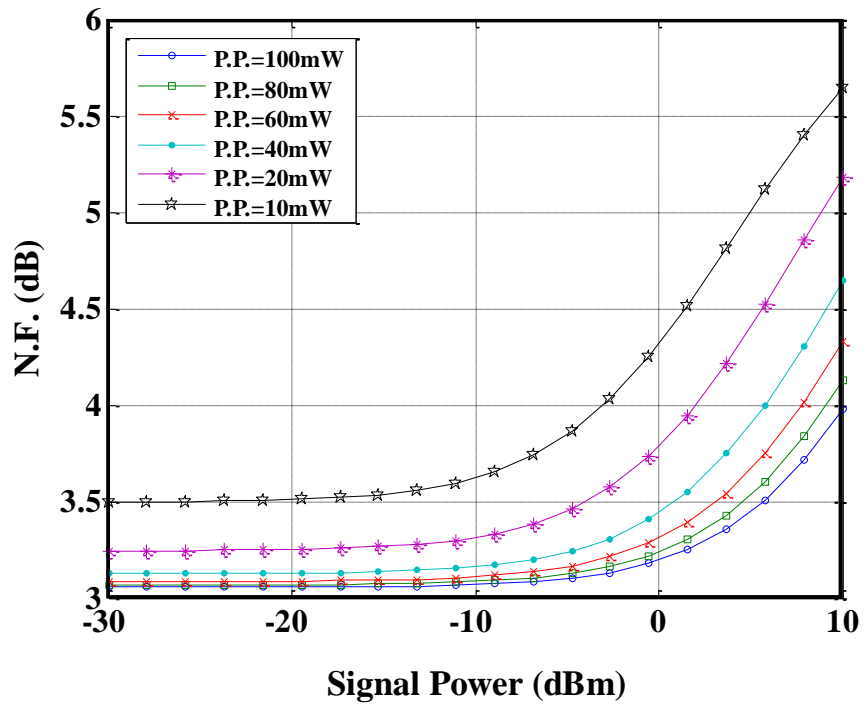

Figure (5): The variation of noise figure versus Signal Power for different pumping power

\section{2- multiple amplified stage}

The effect of multiple amplified stage on the noise figure was tacked into account through this study. Figure (6-a) show the noise figure characteristics as a function of amplified stage number at different fiber length when input power signal, Ion density and pumping power is fixed. This graph was obtained for a seven different fiber lengths $(40,45,50,55,60,65,70) \mathrm{m}$ having $150 \mathrm{ppm}$ doping density and by applying a signal input power of $-30 \mathrm{dBm}$ and pumping power $30 \mathrm{~mW}$. As shown in figure (6-a) noise figure increases significantly with the increasing amplified stage. The reason of that is to continuation amplify to the noise that result from previous amplified stage. As can be shown, 
the noise figure increases with increasing fiber length due to increases the backwards ASE significantly, reducing the inversion level ${ }^{[3,9]}$.

It can be seen from the Figure (6-a, 6-b, 6-c) that the noise figure decreases with increasing pump power therefore the higher pump powers are required to obtain successively lower noise figures for a given gain.
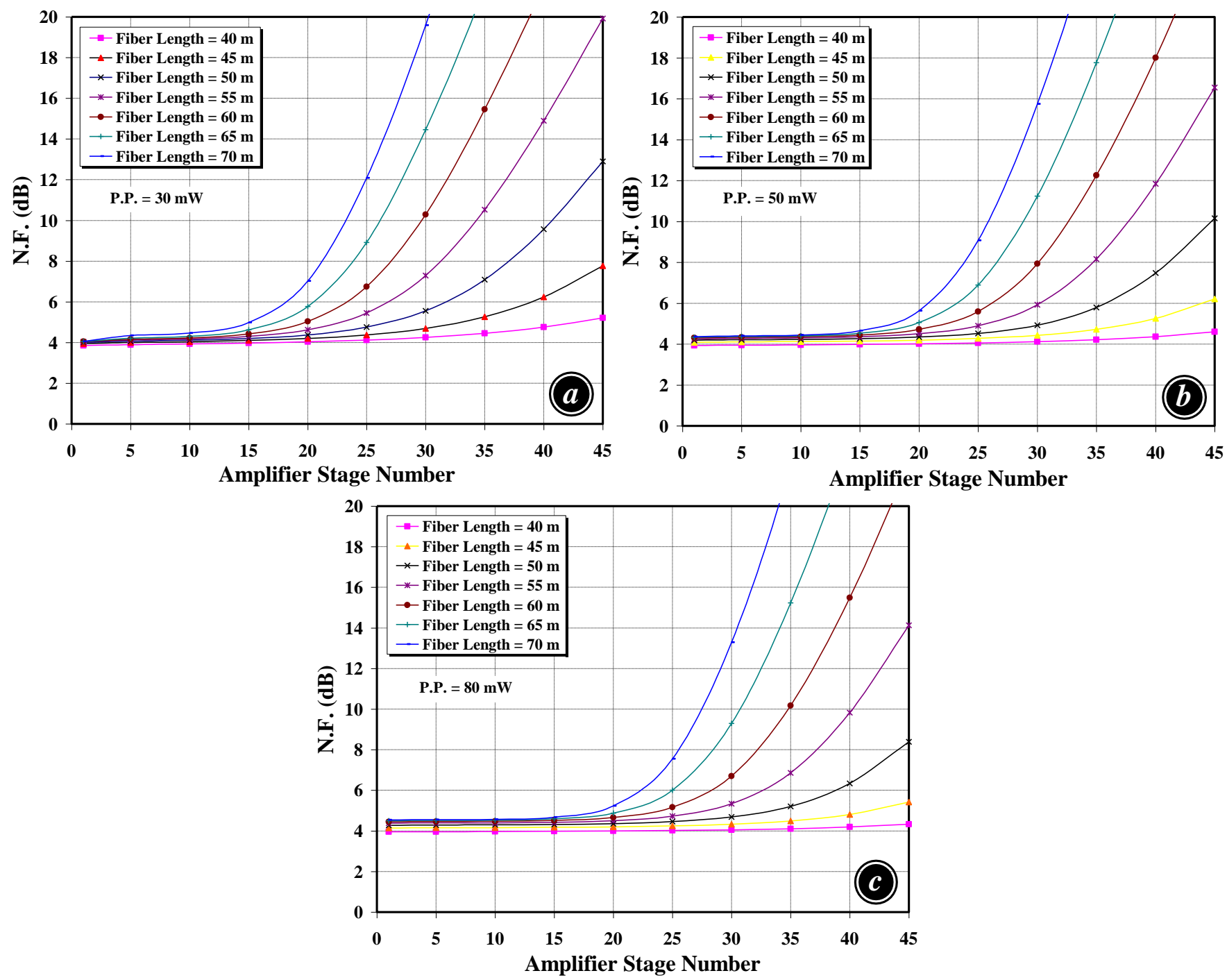

Figure (6) The variation of Noise Figure versus Amplifier Stage Number at a- pumping power $30 \mathrm{~mW}$, b- pumping power $50 \mathrm{~mW}$, c- pumping power $80 \mathrm{~mW}$

\section{V- Conclusions}

The rate and propagation equations characterizing an EDFAs operating in $\mathrm{C}$ band and pumped at $980 \mathrm{~nm}$ in forward direction was numerically solved. According to the results, it was seen that the NF is strongly dependent on the fiber length, pumping power, signal input power and erbium ion density. When the EDFAs is supplied with sufficient pump power, it was shown that EDFAs could be operated in saturation regimes leading minimum noise figure. A continuous increasing of the noise figure value was demonstrated by increasing all of 
amplified stage number and fiber length. In general, successively higher pump powers are required to obtain successively lower noise figures for a given gain. The lower noise figures typically require shorter lengths of fiber (to reduce the backwards ASE) and thus higher pump powers, to achieve the same gain.

\section{VI- References}

1) P. C. Becker, N. A. Olsson and J. R. Simpson "Erbium-Doped Fiber Amplifiers Fundamentals and Technology" printed by Lucent Technologies (USA), 1999.

2) P. M. Aljaff, and B. O. Rasheed "Design Optimization for Efficient Erbium-Doped Fiber Amplifiers" World Academy of Science, Engineering and Technology, Vol. 46, 2008.

3) N. Hossain, et al. "A Numerical Analysis of EDFA for Long Haul Optical Fiber Communication System" 4th International Conference: Sciences of Electronic, Technologies of Information and Telecommunications, 2007.

4) R. Tang, P. L. Voss, J. Lasri, P. Devgan, and P. Kumar "Noisefigure limit of fiber-optical parametric amplifiers and wavelength converters" Optical Letters, Vol. 29, No. 20, 2004.

5) C.R. Giles and E. Desurvire "Modelling Erbium-Doped Fiber Amplifiers", Journal of Lightwave Technology Letters, Vol. 9, No. 2, 1991.

6) B. Bouzid "Performance Tendency of Gain and Noise Figure at Different EDFA Configurations" Journal of Electronics and Technology, Vol. 8, No. 1, 2010.

7) P. Q. Bao and L. H. Son "Gain and Noise in Erbium Doped Fiber Amplifier (EDFA) - a Rate Equation Approach (REA)" Communications in Physics, Vol. 14, No. 1, 2004.

8) A. Altuncu, A.S. Siddiqui, A. Ellis, M.A. Newhouse and A.J. Antos "Gain and Noise Figure Characterisation of a $68 \mathrm{~km}$ Long Distributed Erbium Doped Fibre Amplifier" Electronics Letters, Vol.32, No.19, 1996.

9) Y. Yamamoto and T. Mukai, "Fundamental of optical amplifiers," Opt. Quantum Electronics, Vol. QE-21, 1989. 